\title{
Hormigas zompopas (Atta cephalotes) influyen positivamente en la biodiversidad vegetal de bosques húmedos tropicales
}

Javier Ruiz

Proyecto Biodiversidad. Managua, Nicaragua. Correo electrónico:javierruizphd@gmail.com; www.javierruizperez.blogspot.com

Recibido: abril de 2010 / Aceptado: junio de 2010

REALIZAMOS OBSERVACIONES DE LA HISTORIA NATURAL DE LA HORMIGA ZOMPOPA (Atta cephalotes) en el período de 1995 a 2010. Monitoreamos una población de hormigas zompopas en 2003 y realizamos experimentos de campo en 2006 en el bosque húmedo tropical de la Estación Biológica La Unión en Nicaragua. Los nidos de hormiga zompopa fueron mapeados dentro de una parcela permanente de 6.5 hectáreas. Se estimó la composición de especies vegetales en el área del nido de la hormiga, y se realizó un conteo de plántulas dicotiledóneas y plántulas de palmas (monocotiledóneas). Los resultados indican que las hormigas zompopas prefieren defoliar plántulas de especies dicotiledóneas, favoreciendo la regeneración de palmas sobre el nido de las hormigas. Las zompopas favorecen la regeneración de palmas en áreas donde ellas establecen el montículo principal de sus nidos. En el artículo se discuten las implicaciones de estos resultados para la dinámica de la regeneración en bosques húmedos tropicales.

Palabras clave: plántulas / herbivoría / palmas / dicotiledóneas / claros de luz / bosque húmedo tropical / Formicidae

\section{Introducción}

La competición y la depredación son mecanismos que estructuran comunidades biológicas. La pregunta de investigación fundamental en ecología de comunidades es cómo tantas especies coexisten en competición por básicamente los mismos recursos - el ejemplo clásico es el plankton (Hutchinson, 1961). Dado que las especies se encuentran compitiendo por los mismos recursos (luz, agua, nutrientes, etc.), se espera que en bosques húmedos tropicales la competición por recursos dé como resultado la reducción de la abundancia de especies con características de dispersión y colonización desfavorables (Silvertown, Holtier, Johnson \& Dale, 1992). Bajo esta hipótesis se espera que a medida que el proceso de competición 
prosiga, solamente un grupo de especies con características favorables empezará a dominar el bosque. Por el contrario, en una hectárea de bosque en el Amazonas pueden haber más de 280 especies de plantas de madera dura coexistiendo (Valencia, Baslev \& Paz, 1994; Oliveira \& Mori, 1999). Similar riqueza de especies se puede encontrar en el sureste de Nicaragua, donde aproximadamente 240 especies de plantas de madera dura se encuentran presentes en cuatro sitios en 0.90 hectáreas (Granzow-de-la-Cerda, Zamora, Vandermeer \& Boucher, 1997; Vandermeer, Granzow-de-la-Cerda, Boucher, Perfecto \& Ruiz, 2000).

En la presente investigación propongo que en algunas áreas del bosque húmedo tropical del este de Nicaragua, la depredación desproporcionada de árboles por insectos herbívoros es un mecanismo que reduce la riqueza de especies de árboles y promueve la biodiversidad al favorecer a grupos de especies que se encuentran en menor proporción. Este mecanismo podría ser responsable de la gran abundancia y diversidad de plantas en sotobosques de bosques húmedos neotropicales. Los efectos negativos de la especie de hormiga Atta cephalotes sobre las plantas dicotiledóneas pueden explicar este patrón de dominación por palmas en bosques en regeneración. Estas hormigas cortan grandes volúmenes de hojas en el sotobosque y el dosel del bosque, una colonia puede estar constituida por millones de individuos que pueden llegar a desfoliar árboles enteros (Dirzo, 1987).

La hormiga Atta cephalotes es conocida con el nombre común de hormigas cortadoras u hormigas zompopas. Estas hormigas pueden llegar a desfoliar entre 12 y $17 \%$ del porcentaje de hojas producidas en el bosque (Cherrett, 1986). Se especializan en consumir un hongo del tipo Leucocoprinus, al que alimenta con un bolo alimenticio constituido por las hojas cortadas de bosque (Hölldobler \& Wilson, 1990). La interacción biológica entre el hongo y las hormigas es un mutualismo, ya que las hormigas cargan las esporas del hongo en su cuerpo o en el área del cuello, el hongo es fuente de alimento para las hormigas. Las colonias nuevas de las hormigas se forman después de los vuelos nupciales, cuando nacen nuevas reinas y fundan nuevas colonias. Los nidos de las hormigas se caracterizan por poseer un promontorio prominente de hasta $100 \mathrm{mts}^{2}$. El área del nido se encuentra en constante reciclaje de suelo desde niveles inferiores. En estas áreas el suelo es enriquecido por la presencia de detritos y nutrientes del nivel inferior del suelo (Haines, 1975).

En un estudio de campo realizado en la Estación Biológica de la Selva en Costa Rica se abordó la pregunta: ¿en cuánto tiempo las hormigas pueden reciclar un área de aproximadamente 10 hectáreas de sotobosque? Los resultados sugieren que sería necesario un período de 200 a 300 años para que Atta cephalotes remuevan todo el suelo de un bosques húmedo tropical en Costa Rica (Perfecto \& Vandermeer, 1993). Estos resultados indican que las hormigas tienen el papel benéfico de agregar fósforo, detritos y otros nutrientes desde los horizontes inferiores del suelo hacia la superficie. Además, las condiciones creadas por las hormigas cortadoras potencialmente favorecen el crecimiento y la sobrevivencia de plantas que se encuentran cercanas a la colonia. Dado que las hormigas Atta cephalotes desfolian en proporciones abrumadoras a las plantas leñosas éstas podrían estar librando a las palmas de la competición con plantas de especies de madera dura, las cuales presentan características apropiadas de dispersión y colonización.

Se ha considerado que Atta cephalotes tienen un papel importantes en la composición de las especies del bosque (Haines, 1975). Inclusive se les ha llegado a considerar como 
"keystone species" (Fowler, Silva, Forti \& Saes, 1986), aunque existe muy poca información de campo donde se cuantifique el efecto de estas hormigas sobre la composición de especies en bosques húmedos tropicales.

La idea planteada en este artículo es que las hormigas cortadoras podrían estar facilitando las condiciones para la regeneración de palmas en las áreas donde las hormigas establecen sus colonias, como es el caso del bosque de la Estación Biológica La Selva en Costa Rica (J. H. Vandermeer, comunicación personal, 03 de marzo de 2000). En esta investigación se aborda la hipótesis que las hormigas Atta cephalotes reducen la competición de plantas en el sotobosque a nivel local, favoreciendo a las palmas sobre otras plantas. El enfoque de este trabajo es fitocéntrico y se tratar de comprender y predecir cambios en la composición de especies a nivel de plántulas y vástagos.

\section{Metodología}

\subsection{Sitio de estudio}

El estudio se realizó en un bosque húmedo tropical del este de Nicaragua. El sitio es localmente conocido como Estación Biológica La Unión $\left(12^{\circ} 5^{\prime} \mathrm{N} 83^{\circ} 53^{\prime} \mathrm{W}\right)$ y se caracteriza por ser un bosque húmedo tropical. La temperatura media anual en la zona es de $26^{\circ} \mathrm{C}$, la evapotranspiración anual es de 1332 mm (IRENA, 1991). El bosque de la Estación Biológica La Unión se encuentra a media hora de la ciudad de Bluefields y es accesible por medio del Río Caño Negro. El bosque tiene una copa a unos 30 metros de altura, esta copa está compuesta por árboles de gran tamaño que resistieron los vientos del huracán Juana en 1988, por ejemplo Dipteryx oleifera y Hieronima alchornoques. Un segundo estrato se encuentra a unos 20 metros. Este estrato es compuesto por individuos que regeneraron pasando por el estadio plántula y rebrotes de árboles tumbados por el huracán Juana. El estrato se caracteriza por ser muy compacto, se formó alrededor de 2001. Para 1994 las especies dicotiledóneas pioneras típicas como Ochroma pyramidale y Cecropia sp. se encontraban experimentando altas tasas de mortalidad, un producto de la competición por la luz. Aunque en años recientes hemos hipotetisado que tendría que haber una alta tasa de mortalidad en esta copa, no se han notado mortalidades masivas que pudieran atribuirse a la competición ecológica (Ruiz, Vandermeer, Granzow-de-la-Cerda, Perfecto \& Boucher, 2008). Lo que ocurre en esta copa es que los árboles se encuentran llenando micro-claros de luz que se forman cuando cae una rama (Ruiz, Vandermeer, \& Granzow-de-la-Cerda, 2010). De esta forma los árboles se encuentran coexistiendo, posiblemente contribuyendo a una reducción de la competición ecológica en la copa del bosque. El tercer estrato es el sotobosque, caracterizado por la presencia de plántulas y vástagos de especies leñosas y palmas, aunque algunas especies de palma se encuentran también en el dosel intermedio.

\subsection{Localización y estimación de las características de nidos de Atta cephalotes}

Dentro de la parcela permanente localizamos todos los nidos de la hormiga Atta cephalotes en febrero de 2003. Una etiqueta alumínica con un número único fue colocada en un banderín plástico pinchado al suelo en el centro del nido. Las colonias de hormigas fueron clasificadas en nidos activos e inactivos. En el caso de las colonias activas la presencia de hormigas viviendo en el nido fue el criterio para clasificarlas como activas. Para clasificar una colonia como inactiva constatamos que las entradas y el nido no estuvieran utilizados por las 
hormigas. Se procedió a medir el área del nido de las hormigas definido como el área del sotobosque donde las hormigas se encuentran removiendo el suelo del bosque. Para realizar esta actividad, una persona se colocó al centro del nido y cuatro personas se colocaron a lo largo y ancho del nido. Se midió el largo y el ancho del nido con una cita métrica desde el centro del nido. Se utilizó la forma de una elipse para estimar el área del montículo. En el campo fue fácil determinar los límites del nido para las colonias activas y también para los nidos inactivos puesto que estas áreas sobresalían al nivel del sotobosque.

\subsection{Conteo e identificación de plántulas sobre el nido de las hormigas Atta cephalotes}

Se procedió a identificar las plantas como dicotiledóneas y monocotiledóneas, y a contar el número de plántulas dentro del nido de Atta cephalotes. La identificación taxonómica de plántulas a nivel de especies fue prácticamente difícil al no existir una metodología para realizar la identificación de plántulas a nivel de especie. Por esta razón, utilizamos la categoría de monocotiledóneas y dicotiledóneas. Debido a que las plantas fueron defoliadas por las hormigas Atta cephalotes rápidamente, no fue posible realizar estudios demográficos de las poblaciones de plantas. Otras características de los nidos fueron notadas: el área descubierta (suelo desnudo), el área con hojarasca, área cubierta por monocotiledóneas y dicotiledóneas. Las estimaciones de estas áreas se realizaron visualmente por la misma persona. Dado que el número de colonias inactivas fue mayor que el número de colonias activas, fue necesario llevar a cabo un proceso de selección antes de realizar los análisis estadísticos. El criterio principal para la selección fue que las colonias tuvieran áreas similares. Una vez que se logró tener dos grupos con igual número de colonias inactivas y activas que ocuparan similar área del sotobosque, se procedió a realizar los análisis estadísticos. De esta forma se garantizó que los resultados fueran comparables entre las tres condiciones de sotobosque (sombra, colonias activas y colonias inactivas).

\subsection{Experimento de preferencia de monocotiledóneas y de dicotiledóneas}

Con el objetivo de determinar la preferencia de las hormigas zompopas por plantas dicotiledóneas o monocotiledóneas se seleccionaron cinco colonias activas y se les colocó una porción de hojas en el área de entrada principal en febrero de 2006. El experimento consta de dos tratamientos: el primero es de 40 hojas de dicotiledóneas seleccionadas al azar del dosel del bosque y un volumen similar de hojas de palmas, las que también fueron seleccionadas al azar ente las especies de palmas dominantes en el bosque. Las hojas de dicotiledóneas fueron mezcladas en una bolsa plástica y las hojas de monocotiledóneas fueron mezcladas en otra bolsa plástica. A cada colonia se le aplicaron cinco replicas de cada uno de los dos tratamientos en la entrada principal de la colonia. El primer día se aplicó el tratamiento de las dicotiledóneas y un par de días después el tratamiento de las monocotiledóneas. En total se realizaron 25 replicas por cada tratamiento. Los puñados de hojas fueron suministrados a las colonias en la mañana y revisados 24 horas después para determinar el nivel de preferencia de las hormigas por cada categoría de hojas. 


\section{Resultados}

\subsection{Localización de las colonias de Atta cephalotes}

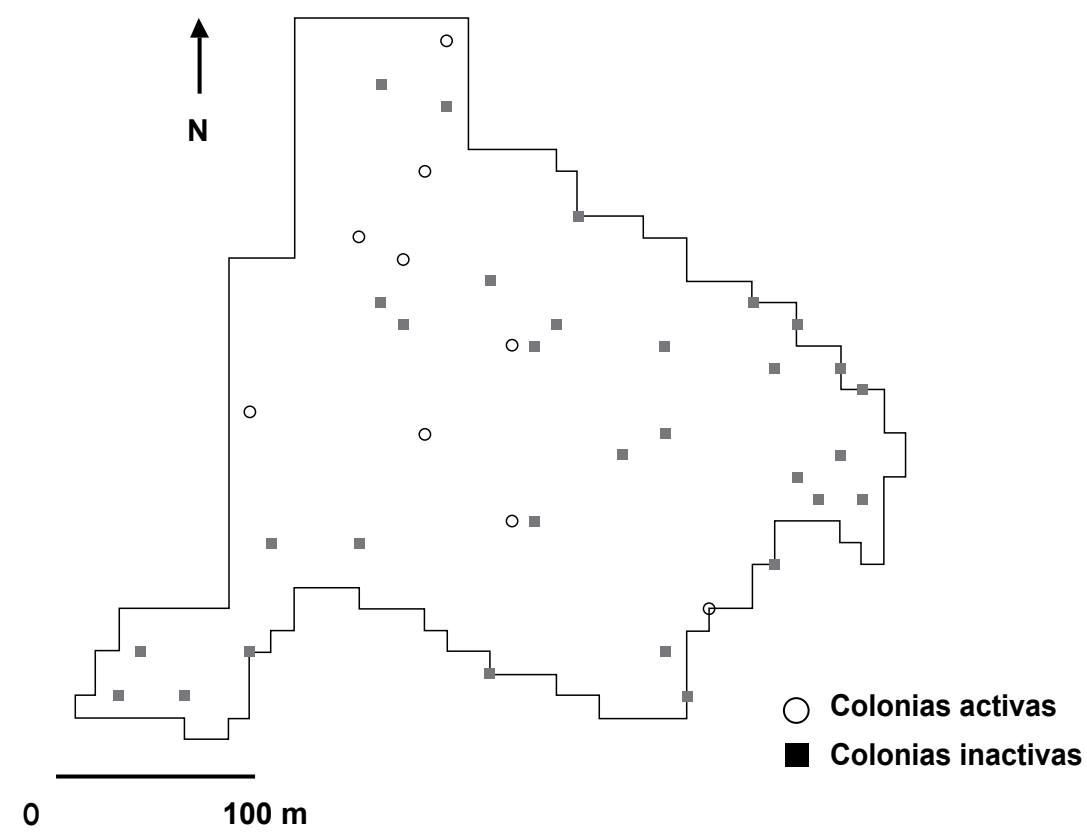

Ilustración 1. Localización de las colonias de Atta Cephalotes en un bosque húmedo tropical del este de Nicaragua. Los cuadros representan colonias inactivas y los círculos representan colonias activas.

En febrero de 2003 se localizó un total de 31 colonias inactivas y 9 colonias activas. El área total que ocupan las colonias inactivas es de $509.3 \mathrm{mts}^{2}$ y el área total que ocupan las colonias activas es de $226.2 \mathrm{mts}^{2}$, para un total de $735.35 \mathrm{mts}^{2}$. Esta área corresponde a un 1.158\% del área total de la parcela de 6.35 ha. La localización de las colonias activas e inactivas se presenta en la ilustración 1. Las colonias activas se encuentran distribuidas en el centro de la parcela. Ésta es el área de mayor altura dentro de la parcela y se mantiene seca la mayor parte del año. Las colonias inactivas se encuentran bordeando la parcela en las áreas de menor altura.

\subsection{Características de las colonias de hormigas activas e inactivas}

La composición de la vegetación sobre los nidos de las hormigas Atta cephalotes depende de la actividad de la colonia de hormigas. En las colonias inactivas las palmas ocupan un 9\% del área del nido y un $22 \%$ corresponde a plántulas. Este $22 \%$ corresponde en su mayoría a plántulas recién germinadas de Simarouba amara, una especie de crecimiento rápido que produce una gran cantidad de semillas a mediados del año. Se ha notado que el período de permanencia sobre el nido de las plántulas de $S$. amara varía entre pocos días a unos cuantos meses. En una investigación de campo se determinó que la sobrevivencia de las 
plántulas de $S$. amara aumenta con la distancia del árbol congénere más cercano en la Estación Biológica La Unión (Sequeira-Cash \& Ingram-Flores, 2006). Otro factor que podría estar limitando el establecimiento de estas plántulas son las condiciones de sequía que ocurren en la estación seca. Se ha observado que las palmas resisten mejor la sequía y en general han estado más tiempo en el sotobosque. El 47\% del área del nido se encuentra cubierto de mucha hojarasca y un $22 \%$ corresponde a suelo desnudo. En las colonias activas, se observa que las palmas ocupan un área considerablemente mayor a la que ocupan las plántulas dicotiledóneas (28\% versus 4\%). Otra característica sobresaliente de las colonias cuando están activas es el porcentaje de suelo desnudo 58\%; solamente un 2\% corresponde a helechos (ilustraciones 2 y 3 ).
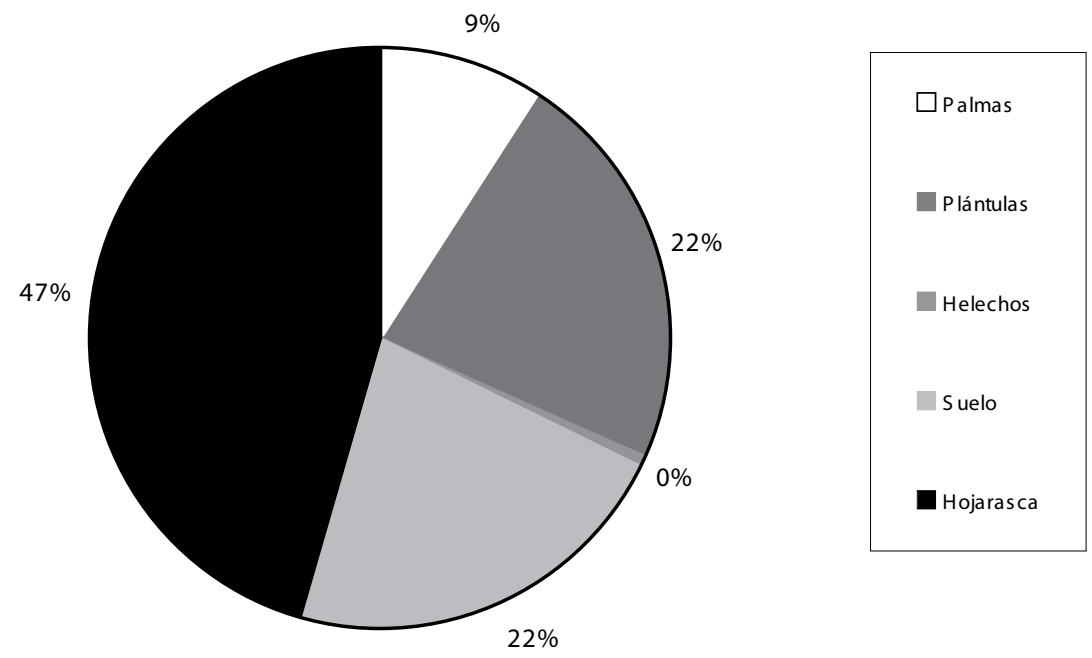

Ilustración 2. Características generales sobre una colonia "inactiva" de la hormiga Atta cephalotes en el bosque húmedo tropical de Nicaragua. Los valores representan el porcentaje del área cubierta por cada categoría.
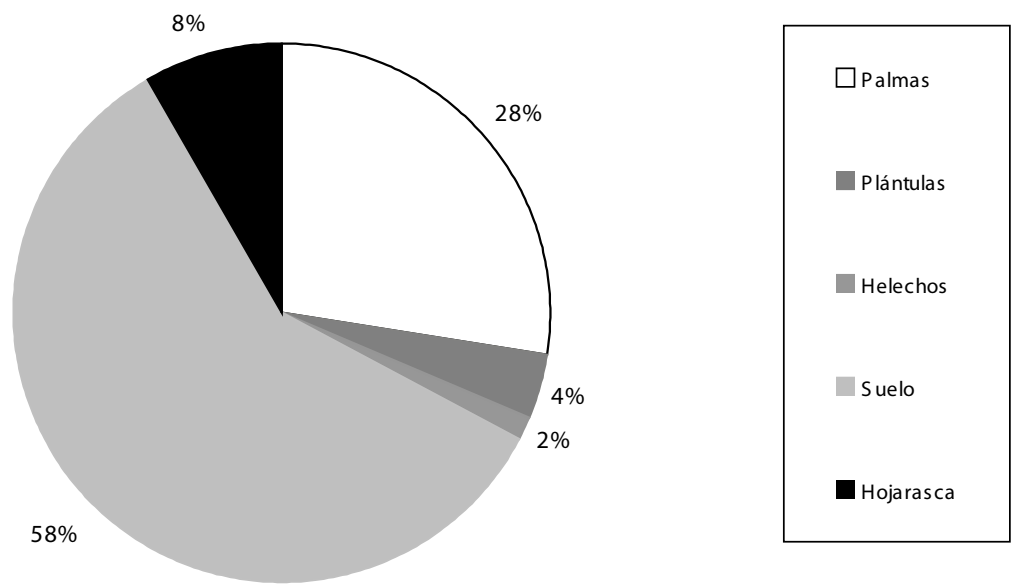

Ilustración 3. Características generales sobre una colonia "activa" de la hormiga Atta cephalotes en el bosque húmedo tropical de Nicaragua. Los valores representan el porcentaje del área cubierta por cada categoría. 


\subsection{Conteo de dicotiledóneas y palmas con estatura $\leq 1$ metro en tres condiciones del sotobosque}

La riqueza de palmas y dicotiledóneas es estadísticamente diferente entre colonias activas, colonias inactivas y el sotobosque sombreado. La tendencia es a que las palmas se encuentren en menor número en las tres condiciones de sotobosque $\left(X^{2}=29.78\right.$, df $=2$, $\mathrm{p}<0.001)$. Se piensa que en el estadio de plántulas las hormigas hubiesen liberado a las palmas antes de la inactividad del nido. Específicamente, el mayor número de individuos en el sotobosque sombreado corresponde a 267 plántulas dicotiledóneas. Este número es de 84 en los nidos activos y tan sólo 34 en nidos inactivos. Por el contrario, la abundancia de palmas es relativamente similar en las tres condiciones (ilustración 4).

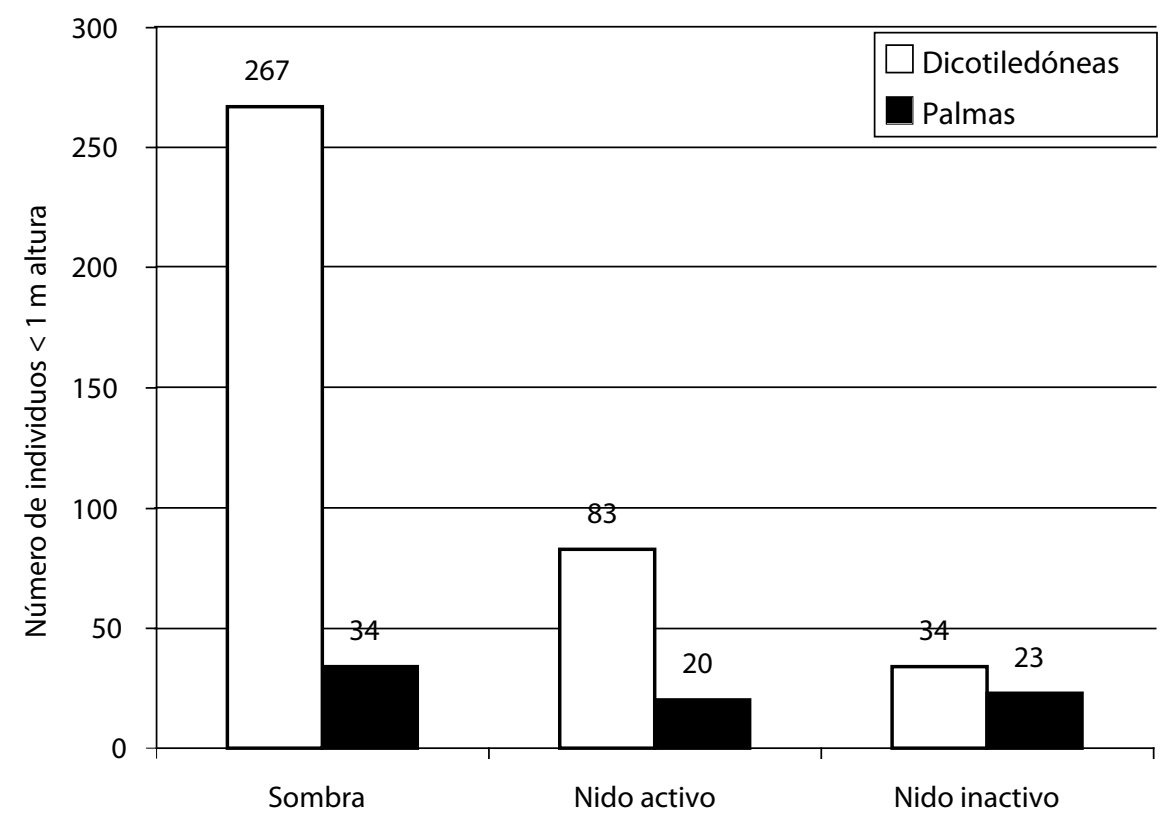

Ilustración 4. Número de plántulas de dicotiledóneas y palmas en tres condiciones en el sotobosque

\subsection{Conteo de dicotiledóneas y palmas (1-2 metros de altura) en tres condiciones del sotobosque}

En el caso de palmas y dicotiledóneas con altura de 1 a 2 metros, existe una diferencia estadísticamente significativa en el número de individuos por cada categoría en las colonias activas, colonias inactivas y el sotobosque sombreado del bosque $\left(X^{2}=, \mathrm{df}=2, \mathrm{p}<0.0001\right)$. La tendencia es que las palmas se encuentran en mayor número que las dicotiledóneas en las áreas de las colonias activas e inactivas, 11 y 22 respectivamente, contra 6 individuos en sotobosque. Las dicotiledóneas se encontraron en mayor número en la sombra (23) y en los nidos activos (40), pero sólo se encontraron 11 individuos contra 22 individuos de dicotiledóneas en los nidos inactivos (ilustración 5). 


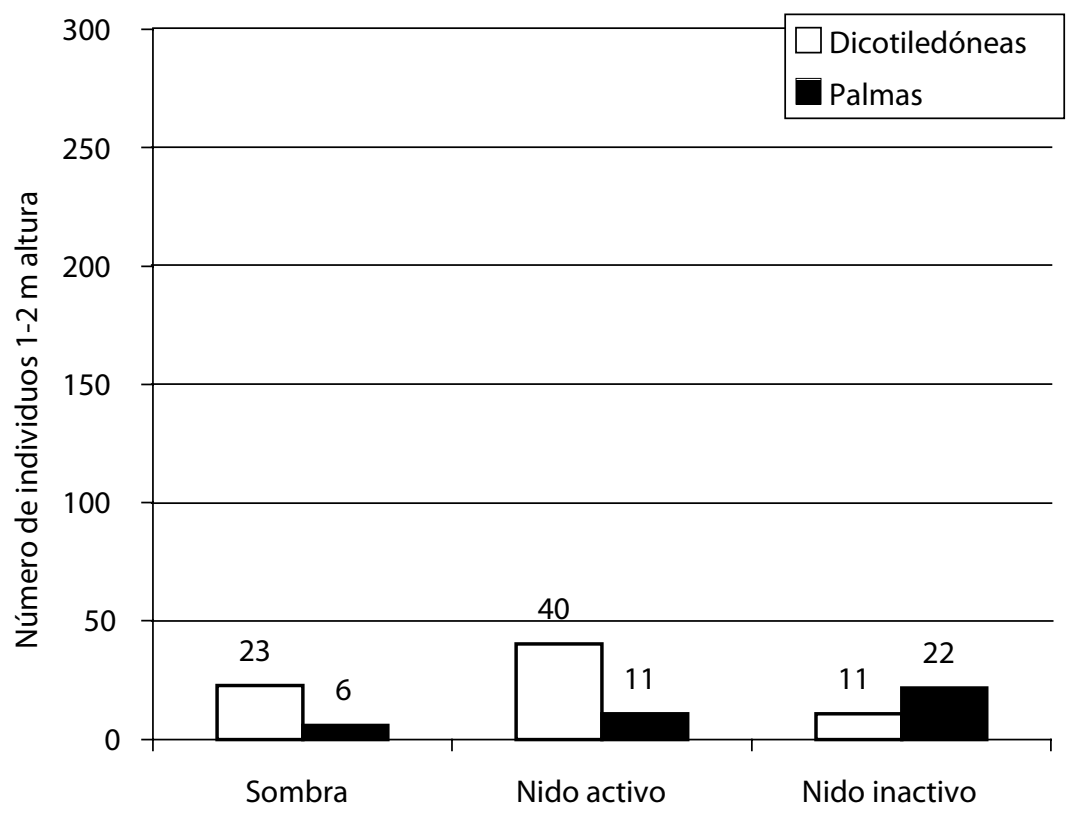

Ilustración 5. Número de vástagos en tres condiciones en el sotobosque.

\subsection{Experimento de palatabilidad de monocotiledóneas y de dicotiledóneas}

Las hormigas zompopas prefieren plántulas dicotiledóneas. Las hormigas transportaron el total de las hojas de las dicotiledóneas hacia dentro de sus nidos en un período de 24 horas. Las palmas fueron rechazadas en su totalidad por estas hormigas.

\section{Discusión}

\subsection{Micro-sitios de regeneración seguros para plantas}

La teoría ecológica de regeneración de bosques húmedos tropicales centra especial énfasis en la importancia de los sitios de regeneración "seguros" para la regeneración de plantas (Brokaw, 1985; Hubbell, 1999). La idea básica es que cuando un árbol cae en una copa cerrada de un bosque húmedo tropical se crean las condiciones de iluminación y espacio en el sotobosque para que las plántulas puedan crecer y establecerse en la copa del bosque (Dalling \& Hubbell, 2002; Dalling, Muller-Landau, Wright \& Hubbell, 2002). La investigación que presento aquí aborda la idea que los nidos de las colonias de Atta cephalotes podrían ser un sitio de regeneración seguro para plántulas de palmas (Perfecto \& Vandermeer, 1993). La evidencia reportada es en favor de la hipótesis que el papel de las hormigas Atta cephalotes es determinante en la composición de especies en bosques húmedos tropicales (Haines, 1975) y que la preferencia de estas hormigas por desfoliar las plantas dicotiledóneas favorece a las palmas.

Las hormigas zompopas son de los herbívoros principales de los bosques húmedos tropicales. Éstas consumen más masa foliar que cualquier otro grupo taxonómico (incluyendo mamíferos 
y otros insectos herbívoros). La cantidad de hojas cortadas por las hormigas zompopas se ha estimado en 12 y 17\% de la producción de hojas (Cherrett, 1986). Esta herbivoría está concentrada en los nidos de las hormigas. Aunque muchos árboles crean claros cuando caen, las hormigas zompopas crean condiciones de alta iluminación sobre sus nidos en el cual se encuentra un bajo número de plántulas regenerando en ellos. En un estudio de campo realizado en la Estación Biológica de La Selva se reportó una baja sobrevivencia de plántulas en los nidos de hormigas zompopas (Haines, 1975), un producto de la alta herbivoría causada por Atta cephalotes a las plantas. Lo que notamos en esta investigación de campo es que los nidos son un micro-sitio de regeneración seguro para las palmas. Lo que hace fascinantes los resultados presentados es el hecho de que las hormigas zompopas están favoreciendo a las palmas de forma tal que las palmas tienes mejores condiciones para regenerar sobre los nidos de hormigas, los que presentan condiciones apropiadas para alcanzar los niveles superiores del dosel del bosque.

Una tasa positiva de crecimiento poblacional de Atta cephalotes podría dar como resultado una abundancia de palmas mayor a la esperada en la sombra de un bosque húmedo tropical. El período de sobrevivencia de un nido de hormiga zompopa ha sido estimado en aproximadamente 16 años. Este período de sobrevivencia, asociado a una tasa de crecimiento poblacional de 0.46 observada en la población de Atta cephalotes en Costa Rica, podría dar como resultado que en 200 a 300 años todo el sotobosque hubiera tenido al menos un nido de hormiga zomopopa en cada punto del bosque (Perfecto \& Vandermeer, 1993). Asociando estas estimaciones a nuestras observaciones de campo, se puede especular que el dominio de las palmas en algunos sitios del bosque húmedo tropical del este de Nicaragua, podría estar relacionado al crecimiento poblacional positivo de las hormigas zompopas.

Otros factores que podrían determinar la tasa de establecimiento de nidos deben tomarse en cuenta. En el estudio de campo realizado por Perfecto y Vandermeer (1993), se determinó que no existe relación entre la densidad de nidos y el tipo de suelo (aluvial versus residual). Aunque aparentemente existe una relación entre el tipo de suelo y la mortalidad y sobrevivencia de las colonias, en nuestro sitio en Nicaragua observamos que podría existir una relación entre la distribución de los nidos y la altura del terreno. Los nidos de hormigas que se encuentran en áreas bajas son propensos a inundarse durante el período lluvioso, presumiblemente reduciendo el período de vida de (o residencia en) la colonia.

Existe una diferencia en la tasa de formación de nuevas colonias entre nuestro sitio y el sitio en el estudio realizado en Costa Rica. Mientras en el bosque de la Estación Biológica de La Selva se observaron formaciones de nuevas colonias, en Nicaragua no observamos formaciones de nuevas colonias durante el período de estudio. Las colonias en Nicaragua incrementaron en actividad, área del nido y posiblemente en la cantidad de material foliar cortado (observación personal). Aun así no se observó la formación de nuevos nidos, aunque sí observamos varias reinas de Atta cephalotes durante un período de 16 años de observaciones de campo (1995-2010). Las razones por las cuales se observa esta baja tasa de establecimiento de nidos de hormigas podrían estar relacionadas a factores como la competición entre colonias congéneres, ataques de armadillos, parásitos del hongo mutualista, etc. 


\subsection{Composición de especies sobre el nido de hormigas Atta cephalotes}

En la ilustración 6 se presenta una representación esquemática de las transformaciones en la arquitectura vertical y composición vegetal de un bosque húmedo tropical durante el período de vida de una colonia de hormigas zompopas. Las ideas presentadas en este esquema surgen de las observaciones de la historia natural de las colonias de las hormigas Atta cephalotes durante el período de 1995 a 2010: A) Primero se observan tres estratos del bosque, un dosel superior, el dosel intermedio y la regeneración avanzada compuesta por plántulas; B) Durante el período de formación de la colonia se observa un pequeño montículo. Sobre éste las hormigas defolian desproporcionadamente a las especies dicotiledóneas. Las hormigas no defolian a las palmas; C) El efecto de las colonias activas sobre la composición de la vegetación es muy marcado al defoliar a las plantas dicotiledóneas. Vandermeer (1990) notó que las condiciones de los claros de luz de gran tamaño generalmente se caracterizan por ser tomados por malezas con las cuales las plantas tienen que competir por las condiciones de luz y espacio presentes en el claro. Los nidos de hormigas Atta cephalotes son diferentes puesto que las hormigas se encargan de defoliar a dicotiledóneas y malezas; D) Cuando las colonias de las hormigas se encuentran inactivas se puede notar un cambio en el tipo de vegetación, en el cual se observan varias palmas. Se ha notado que cuando las colonias están inactivas tienen un suelo con una estructura muy plástica y pegajosa, lo cual hace que el reclutamiento de dicotiledóneas y malezas sea difícil. En este punto la regeneración es dominada por las monocotiledóneas.

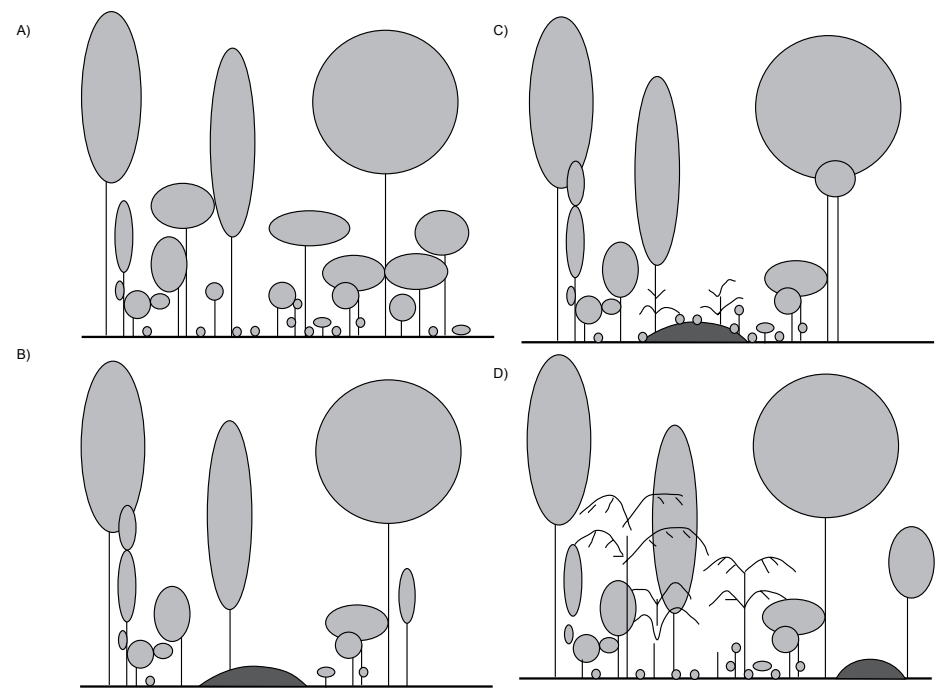

Ilustración 6. Diagrama representando el proceso de formación de las colonias de $A$. cephalotes y la regeneración de especies vegetales sobre dichas colonias: A) Bosque húmedo tropical con tres doseles. B) Formación de colonias, note que las hormigas defolian muchas plantas dicotiledóneas sobre los nidos. Las hormigas defolian mucha vegetación y en ocasiones hasta matan árboles de gran tamaño ubicados al centro de donde se encuentra la colonia establecida. C) Luego de un tiempo las palmas toman ventaja de las condiciones favorables para la regeneración o se dispersan hacia las áreas de las colonias. D) Durante el período de inactividad de la colonia (muerte o migración), las palmas se encuentran en plenas condiciones para crecer hasta el sotobosque. El montículo a la derecha de la figura “D” representa la formación de una nueva colonia de hormigas. 


\subsection{Suposiciones y errores experimentales}

Hay áreas en las que este trabajo podría mejorarse y expandirse. Aunque anualmente se visitaron las colonias al menos una vez, la información de demografía poblacional no está disponible. La razón por la cual no se continuó con el marcaje anual de plántulas sobre las colonias es debido a la alta tasa de mortalidad de plántulas. Esta mortalidad es producto de la defoliación producida por las hormigas, días o semanas después de que la plántula se había etiquetado. Las mediciones de la demografía poblacional de palmas no se realizaron sistemáticamente puesto que la composición de las especies de palmas varía marcadamente dentro y entre colonias. De haber tomado los datos de demografía de tantas especies de palmas las comparaciones resultarían sesgadas debido a la variedad de morfologías de las especies de palmas. El porcentaje de área de la colonia cubierta por las plántulas dicotiledóneas y las palmas se tomó como una aproximación del desempeño de estos grupos vegetales. La preferencia de las hormigas Atta cephalotes por las dicotiledóneas podría deberse a que sus hojas son suaves y presentan un alto contenido de agua, a diferencia de las hojas de palmas que son duras y tiene bajo contenido de agua. Similares observaciones se han realizado en un estudio anterior (Dirzo, 1987). Ya que los resultados basados en los datos de la cobertura y los datos del número de especies o palmas son consistentes, esto nos permite tener confianza en los resultados obtenidos. Otro aspecto que hace difícil la clasificación de las especies sobre las colonias de hormigas es la falta de guías de clasificación taxonómica de campo para especies de bosque húmedo. Se podrían haber realizado ensayos de transplantes y crecimiento para realizar identificaciones taxonómicas a las plántulas y palmas. Aunque esta opción se manejó desde el inicio del estudio, no se llevó a cabo por motivos ajenos a la verificación técnica. La identificación de las colonias activas e inactivas se realizó adecuadamente, aunque no se logró determinar el rango de acción de las colonias de hormigas. La presente investigación se concentra en investigar la regeneración de plántulas sobre el área de la colonia donde el efecto de la defoliación de las hormigas es muy marcado. Las hormigas defolian selectivamente a las dicotiledóneas en la cercanía de las colonias. La defoliación en muchas ocasiones crea aberturas en el bosque, lo que aumenta las condiciones de luz en el sotobosque.

\subsection{Proceso de competición ecológica en bosques húmedos tropicales mediado por la actividad de colonias de hormigas Atta cephalotes}

Nuestros resultados muestran que en el proceso de competencia ecológica por regenerarse en el bosque húmedo tropical, las palmas son favorecidas por la depredación de las plántulas dicotiledóneas por las hormigas Atta cephalotes. Un aspecto que merece más atención es el desarrollar modelos cuantitativos para determinar el efecto de las hormigas en la abundancia de palmas en espacio explícito. Desde un punto de vista teórico, el efecto de las hormigas sobre las palmas podría incluirse como un factor que reduzca el número de plántulas dicotiledóneas en un sistema de dos ecuaciones de competición.

Esta investigación presenta resultados que indican que las hormigas Atta cephalotes prefieren consumir plántulas dicotiledónea y no palmas en bosques húmedos tropicales. Como resultado, las especies que se regeneran en los nidos de Atta cephalotes son las palmas, las cuáles no tienen que entrar a una lotería para dispersarse y luego competir 
por las condiciones de luz en los niveles superiores del bosque. Los resultados sugieren un aumento en el número de especies de palmas en las áreas donde las hormigas han establecido sus colonias.

\section{Agradecimientos}

Esta investigación fue posible gracias al apoyo financiero de múltiples becas de la Fundación Nacional de Ciencias otorgadas a John Vandermeer e Iñigo de la Cerda en el período de 1995 a 2006. Agradezco de forma especial a todos los estudiantes de las Universidades de las Regiones Autónomas de Nicaragua y la Universidad Centroamericana que colaboraron en la toma de los datos de campo. En especial mis agradecimientos al grupo de investigadores de El Proyecto Biodiversidad, Bluefields y al personal administrativo del Centro de Documentación e Investigación de la Costa Atlántica de Nicaragua.

\section{Referencias bibliográficas}

Brokaw, N. V. L. (1985). Gap-Phase Regeneration in a Tropical Forest. Ecology (66), 682687.

Cherrett, J. M. (1986). History of the leaf-cutting ant problem. En C.S. Lofgren \& R. K. Vander Meer (Eds.). Fire ants and leaf-cutting ants: biology and management, pp. 10-17. Boulder, Colorado: Westview Press.

Dalling, J. W. \& Hubbell, S. P. (2002). Seed size, growth rate and gap microsite conditions as determinants of recruitment success for pioneer species. Journal of Ecology (90), $557-568$.

Dalling, J. W., Muller-Landau, H. C., Wright, S. J. \& Hubbell, S. P. (2002). Role of dispersal in the recruitment limitation of neotropical pioneer species. Journal of Ecology (90), 714-727.

Dirzo, R. (1987). Estudio sobre interacciones planta herbívoros en Los “Tuxtlas" Veracruz. Revista Biología Tropical (35), 119-132.

Fowler, H. G. V., Da Silva, P., Forti, L. C. \& Saes, N. B. (1986). Population dynamics of leafcutting ants: a brief review. En C. S. Lofgren \& R. K. Vander Meer (Eds.). Fire ants and leaf-cutting ants: biology and management, pp. 123-145. Boulder, Colorado: Westview Press.

Granzow-de-la-Cerda, I., Zamora, N., Vandermeer, J. H. \& Boucher, J. H. (1997). Biodiversidad de especies arbóreas en el bosque tropical húmedo del Caribe Nicaragüense siete años después del huracán Juana. Revista de Biología Tropical (45), 1409-1419.

Haines, B. L. (1975). Impact of leaf-cutting ants on the vegetation development at Barro Colorado Island. En F. B. Golley \& E. Medina (Eds.). Tropical systems: trends in terrestrial and aquatic research, pp. 99-111. New York: Springer-Verlag. New York Inc.

Hölldobler, B. \& Wilson, E. O. (1990). The ants. Cambridge, Massachusetts: Harvard University Press.

Hubbell, S. P. (1999). Tropical tree richness and resource-based niches. Science (285), 1459a.

Hutchinson, G. E. (1961). The paradox of the plankton. American Naturalist (95), 137-145. 
IRENA. (1991). Diagnóstico de los recursos naturales no renovables. En Generalidades sobre la Costa Atlántica. Reporte Interno, Vol II, pp. 9-14.

Oliveira, A. A. \& Mori, S. A. (1999). A central Amazonian terra firme forest. I. High tree species richness on poor soils. Biodivers Conserv (8), 1219-1244.

Perfecto, I. \& Vandermeer, J. (1993). Distribution and turnover rate of a population of Atta cephalotes in a tropical rain forest in Costa Rica. Biotropica (25), 316-321.

Ruiz, J. Vandermeer, J. \& Granzow-de-la-Cerda, I. (2010). Growth and mortality patterns in a thinning canopy of post-hurricane regenerating rainforest in Eastern Nicaragua (19902005). Revista de Biología Tropical. En prensa.

Ruiz, J., Vandermeer, J., Granzow-de-la-Cerda, I., Perfecto, I. \& Boucher, H. D. (2008). Regeneración de bosques huracanados de Nicaragua (1988-2007). Wani (52), 6-16.

Sequeira-Cash, K.\&Ingram-Flores, C.L. (2006).Efecto de la distancia del árbol reproductor en la abundancia y crecimiento de plántulas de Simarouba amara en Fine Pine Wood. Póster presentado durante la Jornada Científica Anual de Universidades de las Regiones Autónomas de la Costa Caribe Nicaragüense. (URACCAN). 01 de agosto de 2006, Recinto URACCAN-Bluefields.

Silvertown, J., Holtier, S., Johnson, J. \& Dale, P. (1992). Cellular automaton models of interspecific competition for space - the effect of pattern on process. Journal of Ecology (80), 527-534.

Valencia, R., Balslev, H. \& Paz, G. (1994). High tree alpha diversity in Amazonian Ecuador. Biodivers Conserv (3), 21-28.

Vandermeer, J. (1990). Crecimiento y sobrevivencia de plántulas de Welfia georgi en un bosque pluvial en la costa caribeña de Costa Rica. Revista de Biología Tropical (38), 7-20.

Vandermeer, J. H., Granzow-de-la-Cerda, I., Boucher, D. H., Perfecto, I. \& Ruiz, J. (2000). Hurricane disturbance and tropical tree species diversity. Science (290), 788 -791. 